\title{
Femtosecond laser-induced quantum-beat superfluorescence of atomic oxygen in a flame
}

\author{
Pengji Ding $\odot,{ }_{1,2, *}$ Christian Brackmann $\odot,{ }^{2}$ Maria Ruchkina $\odot,{ }^{2}$ Mingyang Zhuzou, ${ }^{1}$ Luojia Wang, ${ }^{3, \dagger}$ Luqi Yuan, ${ }^{3}$ Yi Liu, ${ }^{4}$ \\ Bitao Hu, ${ }^{1, \star}$ and Joakim Bood ${ }^{2}$ \\ ${ }^{1}$ School of Nuclear Science and Technology, Lanzhou University, Lanzhou 730000, China \\ ${ }^{2}$ Division of Combustion Physics, Department of Physics, Lund University, Box 118, SE-221 00 Lund, Sweden \\ ${ }^{3}$ State Key Laboratory of Advanced Optical Communication Systems and Networks, School of Physics and Astronomy, \\ Shanghai Jiao Tong University, Shanghai 200240, China \\ ${ }^{4}$ Shanghai Key Laboratory of Modern Optical System, University of Shanghai for Science and Technology, \\ 516, Jungong Road, Shanghai 200093, China
}

(Received 6 May 2021; accepted 7 September 2021; published 16 September 2021)

\begin{abstract}
Among different approaches to generate mirrorless lasing, resonant multiphoton pumping of gas constituents by deep-UV laser pulses exhibits so far the highest efficiency and produces measurable lasing energies, but the underlying mechanism was not yet fully settled. Here, we report lasing generation from atomic oxygen in a methane-air flame via femtosecond two-photon excitation. Temporal profiles of the lasing pulses were measured for varying concentrations of atomic oxygen, which shows that the peak intensity and time delay of the lasing pulse approximately scales as $N$ and $1 / \sqrt{N}$, respectively, where $N$ represents the concentration. These scaling laws match well with the prediction of oscillatory superfluorescence (SF), indicating that the lasing we observed is essentially SF rather than amplified spontaneous emission. In addition, the quantum-beating effect was also observed in the time-resolved lasing pulse. A theoretical simulation based on nonadiabatic Maxwell-Bloch equations well reproduces the experimental observations of the temporal dynamics of the lasing pulses. These results on fundamentals should be beneficial for the better design and applications of lasing-based techniques.
\end{abstract}

DOI: 10.1103/PhysRevA.104.033517

\section{INTRODUCTION}

Mirrorless lasing, where no resonant cavity is required for generating coherent, directional, laserlike emission, has attracted great attention in the past decade due to its significance in fundamental light-matter interaction research and potential application in remote atmospheric sensing and diagnostics of combustion and reacting flows [1,2]. Two main approaches have succeeded in the generation of backward-propagating lasing. One approach is based on electron impact excitation of neutral nitrogen molecules driven by circularly polarized femtosecond laser pulses during filamentation, resulting in lasing emission at a wavelength of $337 \mathrm{~nm}$ [3-10]. Another approach employs resonant multiphoton excitation of atoms or molecules with deep-ultraviolet (UV) laser pulses [11-25].

The multiphoton-excited lasing effect of atomic species was first observed by Aldén et al. in flames under subatmospheric pressures [11]. Nanosecond 226-nm laser pulses were

\footnotetext{
*dingpj@lzu.edu.cn

†j jwang@sjtu.edu.cn

${ }^{*}$ hubt@1zu.edu.cn
}

Published by the American Physical Society under the terms of the Creative Commons Attribution 4.0 International license. Further distribution of this work must maintain attribution to the author(s) and the published article's title, journal citation, and DOI. Funded by Bibsam. used first to photodissociate molecular oxygen and then to excite the formed atomic oxygen via the $(2 p)^{3} P \rightarrow(3 p)^{3} P$ transition [11]. The excitation establishes a population inversion and therefore results in stimulated emission (SE) at $845 \mathrm{~nm}$ via the $(3 p)^{3} P \rightarrow(3 s)^{3} S$ transition [11]. This pioneering work initiated extensive studies of lasing from other atomic and molecular species, such as $\mathrm{H}$ [12], $\mathrm{C}$ [13], N [14], and $\mathrm{NH}_{3}$ [15], etc., and exploration of the potentials for lasing as a technique for combustion and reactive-flow diagnostics [15-17]. With the same excitation configuration as Aldén et al. [11], Dogariu et al. generated 845-nm backward lasing of atomic oxygen in atmospheric air using sub-mJ picosecond 226-nm laser pulses for excitation [18]. Backward-emitted lasing pulses at the $\mu \mathrm{J}$ level of both atomic oxygen and nitrogen were produced using a powerful prepulse to predissociate the molecules [20], showing the possibility of lasing-based remote atmospheric sensing. Even though more efficient methods and new schemes for lasing generation have been developed, the identification of the underlying mechanism responsible for multiphoton-excited atomic and molecular lasing remains unsettled. Two distinct mechanisms have been proposed: Amplified spontaneous emission (ASE), which is based on population inversion [11-14,18,20], and superfluorescence (SF) which requires both population inversion and atomic coherence in the gain medium $[19,26]$. By analyzing the spiky temporal profile of the 845-nm lasing pulse generated from atomic oxygen by excitation using 10-ns, 226-nm laser pulses, Traverso et al. suggests that nonadiabatic atomic coherence is present in the gain medium and 


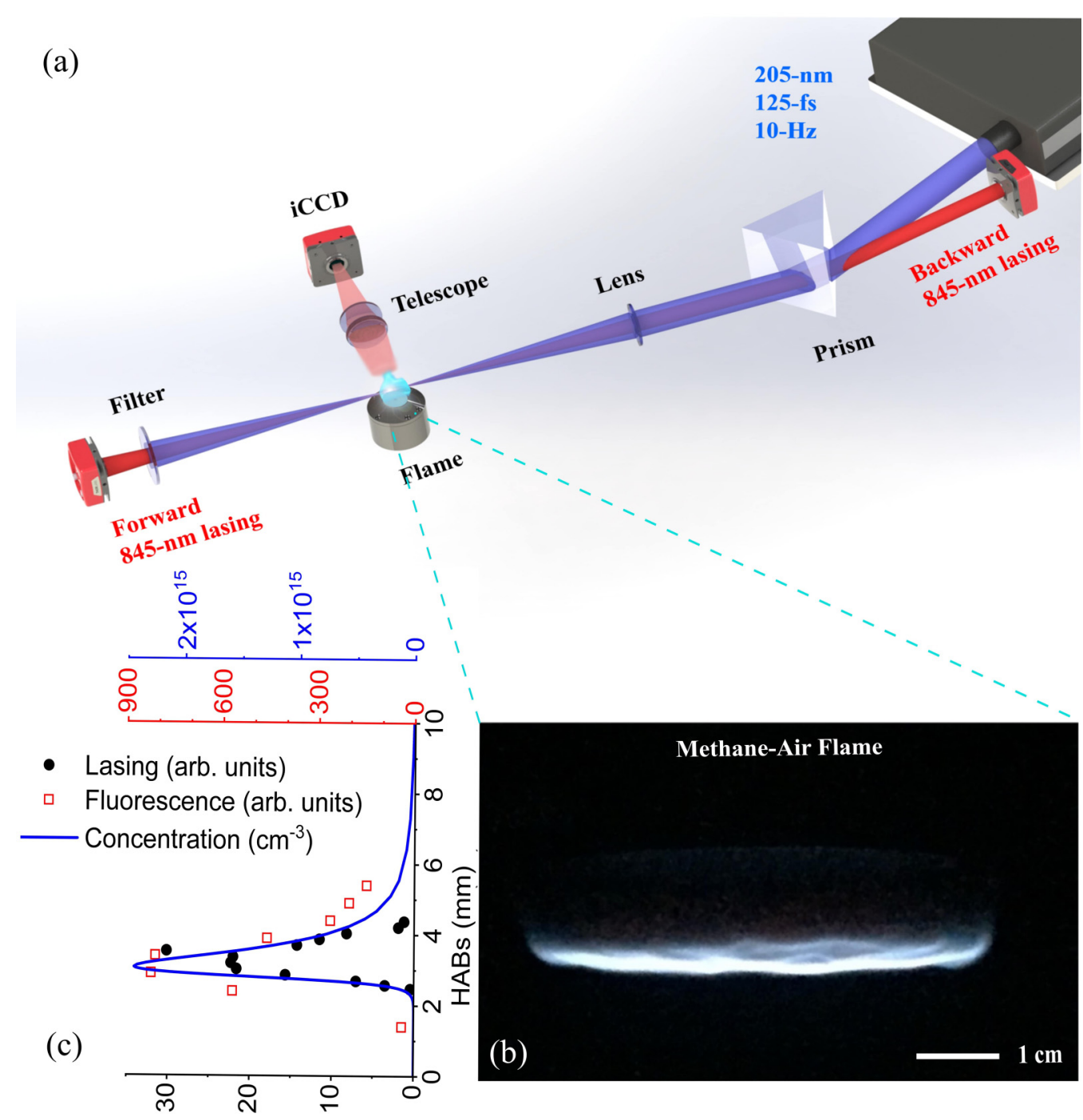

FIG. 1. (a) Schematic illustration of the experimental setup and lasing process. (b) Chemiluminescence image of the methane-air flame. (c) Measured side fluorescence and forward-lasing signals vs height above burner (HAB) together with the concentration profile of the oxygen atom simulated with CHEMKIN-PRO.

the lasing emission is essentially SF [19]. Since SF relies on numerous radiating dipoles being prepared in phase, it is sensitively affected by collisional dephasing. By changing the gas pressure, Dogariu et al. tested the influence of the dephasing collision frequency on the temporal dynamics of the lasing pulse and found it to be independent of gas pressure [1]. Based on this observation, the authors conclude that multiphoton excited lasing is associated with stimulated emission rather than SF. In these experiments, the 10-ns laser pulses serve for both predissociating the molecules and resonantly pumping the resultant atoms. Hence, the subsequent occurrence of these two different processes complicates the determination of the lasing mechanism. Additionally, the methods for identifying the lasing mechanism, such as measuring the lasing energy as a function of the pump pulse energy, are indirect.

In this paper, we employ an ultrashort $(\sim 125 \mathrm{fs})$ laser pulse to generate 845 -nm lasing of atomic oxygen in a wellcharacterized flame, where atomic oxygen is naturally present and its concentration can be well quantified. While Rahman et al. investigated a very similar experimental scenario, they were limited to excitation energies of less than $20 \mu \mathrm{J}$ and could not observe lasing action [27]. As a direct observation of the lasing mechanism, here we measure the dependence of the temporal dynamics of the lasing pulse on the O-atom concentration with an ultrafast streak camera.

\section{EXPERIMENTAL METHODS}

Figure 1(a) shows the schematic of the experimental setup. A wavelength-tunable femtosecond laser system was used to provide 125 -fs laser pulses at $226 \mathrm{~nm}$ with a maximum pulse energy of $\sim 100 \mu \mathrm{J}$. The beam size was about $5 \mathrm{~mm}$ in diameter, and the laser spectral bandwidth was about $2 \mathrm{~nm}$ [full width at half maximum (FWHM)]. The laser beam was first sent through a dispersive prism and then focused with an $f=300 \mathrm{~mm}$ spherical $\mathrm{CaF}_{2}$ lens into a lean methane-air flame. The flame, with an equivalence ratio of 0.51 and a total reactant flow of $15.8 \mathrm{slm}$, was prepared on a $60-\mathrm{mm}$-diameter porous-plug burner. The flame was lifted about $2 \mathrm{~mm}$ above the burner surface and was stabilized with a steel stagnation 

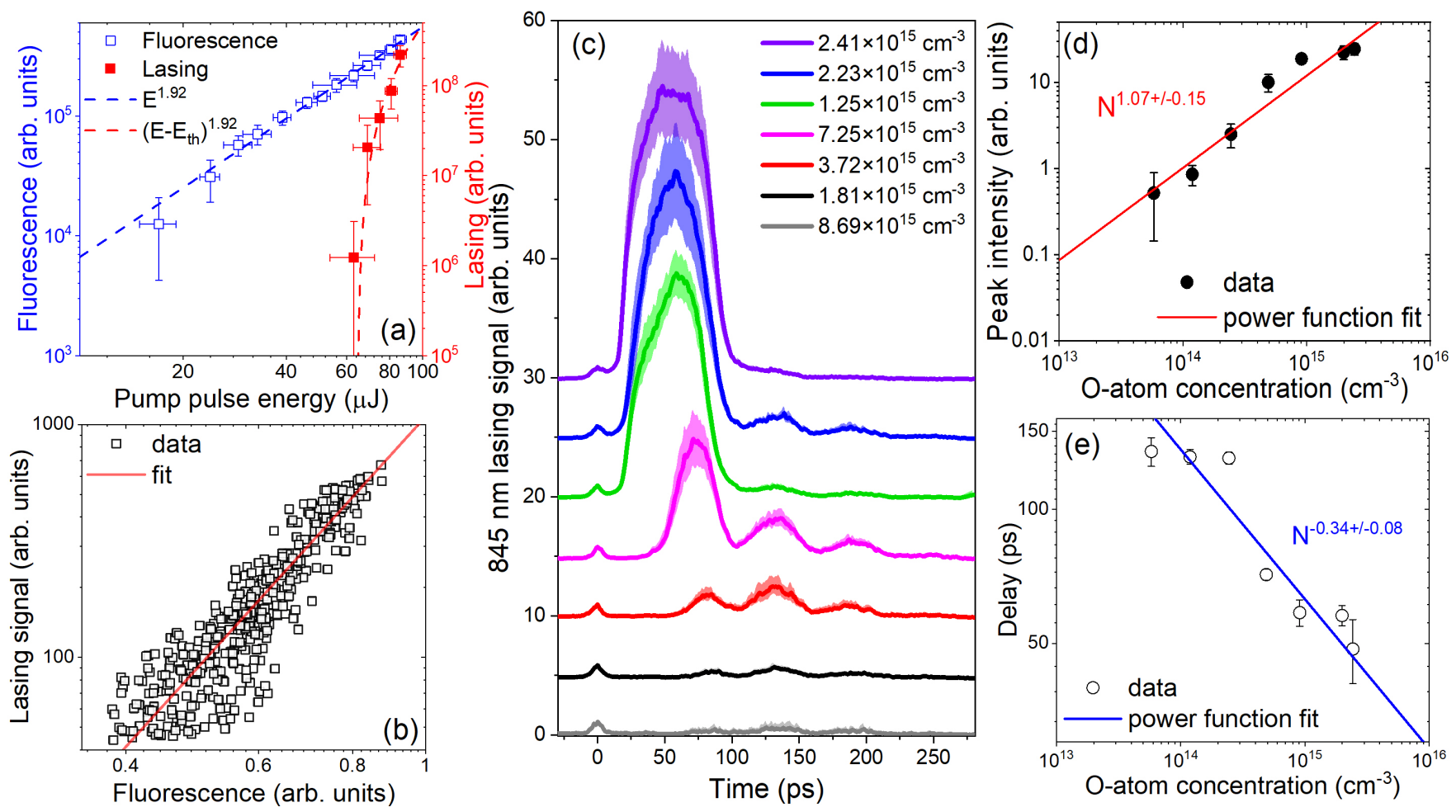

FIG. 2. (a) The forward on-axis and side off-axis 845-nm emission signals as a function of pump pulse energies showing the difference between coherent lasing and incoherent fluorescence. Each data point was averaged for 100 shots and the error bars indicate one standard deviation. (b) The forward on-axis signal vs the side off-axis signal suggests strong optical gain. (c) Time-resolved 845-nm lasing pulses of atomic oxygen at different concentrations ranging from $8.7 \times 10^{13}$ to $2.4 \times 10^{15} \mathrm{~cm}^{-3}$. The peak at 0 ps represents the $226-\mathrm{nm}$ pump pulse. Each profile with an error bar superimposed was averaged five times. (d), (e) The peak intensity and time delay of the first peak of lasing pulse as a function of the concentration. Both curves are fitted with the power function, which suggests a power index of $1.07 \pm 0.15$ for the peak intensity and $-0.34 \pm 0.08$ for the time delay.

plate on the top. For this type of flame the species concentration is constant in the horizontal direction across the burner and only varies in the vertical direction in the flame [28].

Focusing the 226-nm laser pulses into the flame creates a narrow pencil-shaped volume of excited $\mathrm{O}$ atoms, from where 845-nm emission with a narrow linewidth (FWHM $0.07 \mathrm{~nm}$ ) occurs in both the forward and backward directions. The backward emission beam is collimated by the focusing lens, and then separated from the incident 226-nm laser beam by a dispersive prism. Both the forward and backward emission signals are detected with an intensified CCD camera (iCCD, Princeton Instruments, PI-MAX4 1024f), or a streak camera (Opto-Scope S20, Optronis) with a temporal resolution of 2 ps, and a fiber spectrometer (Flame UV-NIR, Ocean Optics). Another iCCD camera, equipped with a $135-\mathrm{mm}$, $f / 2.8$ lens (Nikon), was used to image the 845 -nm fluorescence of atomic oxygen emitted in the side direction.

\section{RESULTS AND DISCUSSIONS}

Figure 2(a) shows the pump pulse energy dependence of the 845-nm emission collected in the forward direction and the fluorescence collected in the side direction. As can be clearly seen, the scalings of the two plots are distinctly different. The forward emission signal exhibits a much sharper dependence on the pump laser pulse energy than the side fluorescence. Also, it shows a threshold signature, indicating that the forward emission is gain dominated. Power-function fitting using $S=a \times E^{b}$ to the data suggests an exponent of $1.92 \pm$ 0.05 for the side fluorescence. For the forward emission, an additional parameter $E_{\text {th }}$ representing the lasing threshold was introduced to the power function, i.e., $S=a \times\left(E-E_{\mathrm{th}}\right)^{b}$, and the fitting to the data suggests an exponent of $1.92 \pm 0.49$ and a threshold of $64 \mu \mathrm{J}$. Both exponents are well in line with two-photon excitation as expected. Furthermore, a direct comparison between the forward emission and side fluorescence shows a nonlinear dependence with a power index of 3.5, as can be seen in Fig. 2(b). The forward emission collected in less than 0.012 sr solid angle is about 700 times stronger than the integrated fluorescence over $4 \pi$ sr. Moreover, singleshot, far-field images of the forward emission beam were also recorded with the iCCD camera, showing that the beam is well localized, with an average divergence of $5.0 \mathrm{mrad}$, i.e., close to the diffraction-limited value of $4.2 \mathrm{mrad}$ given the $100-\mu \mathrm{m}$ diameter of the gain volume. These results unambiguously suggest that the generated axial 845-nm emission from atomic oxygen is gain-dominated lasing.

In order to further identify the nature of $845-\mathrm{nm}$ lasing emission, i.e., whether the underlying mechanism of this lasing process is ASE or SF, a crucial experiment is to measure the temporal dynamics of the lasing pulses with varying concentrations of the emitters. To estimate the O-atom concentration along the vertical direction, we generated 845-nm lasing pulses at different heights above the burner (HAB) in 


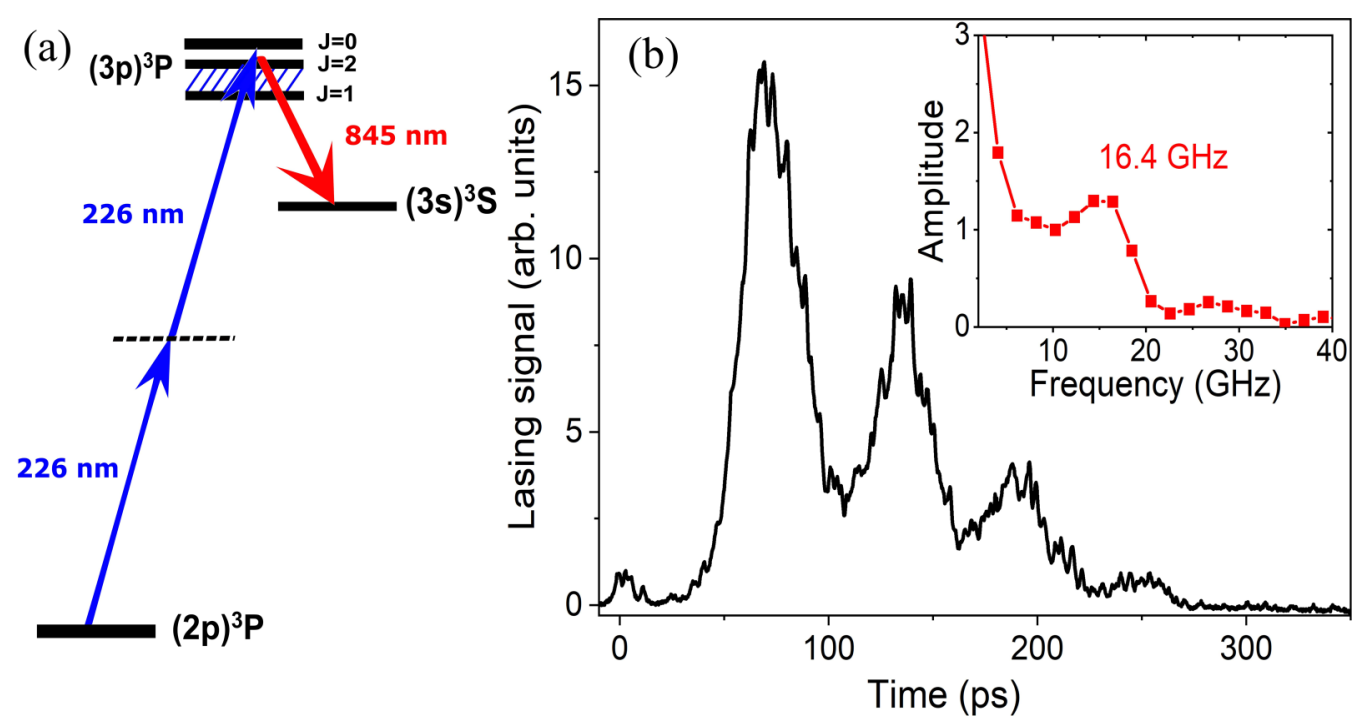

FIG. 3. Quantum-beating effect leads to periodic oscillations of lasing intensity in time. Single-shot, temporal profile of 845-nm lasing pulse measured for an $\mathrm{O}$-atom concentration of $\sim 7.3 \times 10^{14} \mathrm{~cm}^{-3}$. The inset shows the corresponding FFT result, which suggests a oscillation frequency of $16.4 \mathrm{GHz}$.

the flame and the peak intensity of the forward lasing signal corresponds to the solid circles in Fig. 1(c). The corresponding 845-nm fluorescence (open squares), measured from the side, as well as the simulated oxygen concentration profile (solid line), are also plotted in the same diagram for comparison. The concentration profile was simulated using the stagnation plane module in CHEMKIN-PRO with the GRI 3.0 mechanism [29], including multicomponent diffusion transport as well as thermal diffusion. The parameters GRAD $=0.1$ and CURV $=0.5$ resulted in a grid of $\sim 150$ points. As can be noticed, the vertical profiles of the lasing and fluorescence signals deviate from the simulated concentration profile at HABs around $4 \sim 5 \mathrm{~mm}$, but otherwise they match seemingly well within the region of highest concentration. The maximum concentration is estimated to be $\sim 2.50 \times 10^{15} \mathrm{~cm}^{-3}$, located at a HAB of $\sim 3 \mathrm{~mm}$.

Time-resolved measurements were performed for different O-atom concentrations and the results are shown in Fig. 2(c). Time zero is defined as the temporal position of the pump pulse. It is noticed that the lasing pulse exhibits a periodic modulation. In addition the lasing pulse becomes more intense and appears less delayed to the pump pulse with increasing O-atom concentration, exhibiting a typical signature of SF [30]. The intensity and delay time of the first peak is plotted versus concentration in Figs. 2(d) and 2(e). Fitting a power function $y=a \times N^{b}$ to the data, where $N$ is the oxygen-atom concentration, results in a power index of $1.07 \pm 0.15$ for the peak intensity and $-0.34 \pm 0.08$ for the delay time. The fitting errors were estimated from the variation of the reduced chisquare statistic $\chi^{2}$. The oscillatory SF theory predicts that the peak intensity is proportional to $N$, while the delay time is proportional to $1 / \sqrt{N}$ [31-34]. For the data shown in Figs. 2(d) and 2(e) the concentration dependence of the peak intensity agrees well with the $N$ scaling law of oscillatory SF, while the corresponding dependence of the delay time deviates slightly from the predicted $1 / \sqrt{N}$ dependence. This deviation could originate from the uncertainties in determining the locations of the first peaks due to low signal-to-noise ratio in the case of low concentration on the one hand and large temporal width in the case of high concentration on the other hand. To sum up, the agreement on scaling laws indicates that the lasing emission from atomic oxygen is essentially SF.

The observed temporal oscillation on the $845-\mathrm{nm}$ SF pulse is a result of quantum beating between fine-structure transitions of the oxygen atom. The emission line at $845 \mathrm{~nm}$, corresponding to the $(3 p)^{3} P \rightarrow(3 s)^{3} S$ transition, is composed of three finely separated lines $(3 p)^{3} P_{0},(3 p)^{3} P_{2}$, and $(3 p)^{3} P_{1} \rightarrow(3 s)^{3} S_{1}(844.625,844.636$, and $844.676 \mathrm{~nm}$, respectively) due to the interaction between orbital angular momenta and spin [see Fig. 3(a)] [27,35]. The central wavelength separations of the lasing lines are 0.011, 0.040, and $0.051 \mathrm{~nm}$, respectively, which is beyond the resolution of our spectrometer. If the $\mathrm{O}$ atoms are promoted by 226-nm laser excitation into a coherent superposition of all three $J$ states, quantum beating is expected at the frequencies 4.62 , 16.82, and $21.45 \mathrm{GHz}$, respectively. Figure 3(b) shows a typical single-shot temporal profile of the SF pulse pumped with an optimum laser wavelength of $225.6 \mathrm{~nm}$ for excitation. Fast Fourier transformation (FFT) of the result gives an oscillation frequency peak at $16.40 \mathrm{GHz}$, which matches well with the theoretical value of $16.82 \mathrm{GHz}$. This means, under our experimental conditions, that the excitation using 226-nm laser pulses promoted the $\mathrm{O}$ atom into a coherent superposition of the $(3 p)^{3} P_{1}$ and $(3 p)^{3} P_{2}$ state whereas the highest $(3 p)^{3} P_{0}$ state was not involved in the process. We note that most of the $\mathrm{O}$ atoms in the ground $(2 p)^{3} P_{1}$ state reside in the $J^{\prime}=1,2$ states at the flame temperature, according to the work by van Gessel et al. [36]. As a result, the most efficient excitation paths using the 226-nm laser pulse are $J^{\prime}=1 \rightarrow J=1$ and $J^{\prime}=2 \rightarrow J=2$, i.e., most of the excited $\mathrm{O}$ atoms reside in $(3 p)^{3} P_{1}$ and $(3 p)^{3} P_{2}$ states, which perfectly explains our observation that only one quantum-beat frequency was observed. In addition, it needs to be emphasized that the observed temporal oscillation differs fundamentally from the commonly reported temporal ringing in pure SF that 
occurs when the lasing field is reabsorbed and reemitted during propagation through a long cylindrical excitation volume $[31,32]$.

To simulate the experimental results of $\mathrm{O}$-atom $\mathrm{SF}$ and the accompanying quantum-beat phenomena, we use a fourlevel diagram to model the atomic oxygen and numerically solve the Maxwell-Bloch equations. We considered a onedimensional pencil-like medium of $4 \mathrm{~mm}$ length, mimicking the experimental condition. The two upper levels of atomic oxygen $a_{1}$ and $a_{2}$, spaced by $\omega_{21}=2 \pi \times 16.8 \mathrm{GHz}$, are pumped from the ground level $c$ by a 125-fs laser pulse centered at $226 \mathrm{~nm}$ via a two-photon process, producing the emission centered at $845 \mathrm{~nm}$ and another pulse at $130 \mathrm{~nm}$ from the transition $b \leftrightarrow c$. Using the rotating-wave approximation (RWA) and slowly varying approximation, the Hamiltonian in the interaction picture reads [37]

$$
\begin{aligned}
H= & \hbar \delta_{1}\left|a_{1}\right\rangle\left\langle a_{1}\left|+\hbar\left(\omega_{21}+\delta_{1}\right)\right| a_{2}\right\rangle\left\langle a_{2}\right| \\
& +\left[-\left(\hbar \Omega_{p l} e^{i \Delta t}+\hbar \Omega_{b c}\right)|b\rangle\langle c|\right. \\
& \left.-\left(\hbar \Omega_{p u} e^{i \Delta t}+\hbar \Omega_{a b}\right)\left(\left|a_{1}\right\rangle\left\langle b|+| a_{2}\right\rangle\langle b|\right)+\text { H.c. }\right] .
\end{aligned}
$$

Here, $\Omega_{p u}=\wp_{a b} E_{p} / \hbar$ and $\Omega_{p l}=\wp_{b c} E_{p} / \hbar$ are pump Rabi frequencies for the upper and lower transitions, with $E_{p}$ being the amplitude of the pump field. The $a_{1} \leftrightarrow b$ and $a_{2} \leftrightarrow b$ transitions are assumed to have the same dipole moment $\wp_{a b}$, and $\wp_{b c}$ is the dipole moment of the $b \leftrightarrow c$ transition. $\Omega_{a b}$ and $\Omega_{b c}$ are slowly varying Rabi frequencies of the generated fields from the upper and lower transitions, respectively. The detunings are given by $\Delta=\omega_{b c}-v_{p}$ and $\delta_{1}=\omega_{a_{1} c}-2 v_{p}$, where $v_{p}$ is the pump laser frequency. To obtain the Maxwell-Bloch equations for the generated fields, the coherence can be expressed as a sum of slow and fast varying terms $\rho_{a_{i} b}=\sigma_{a_{i} b}+u_{a_{i} b} e^{-i \Delta t}$ and $\rho_{b c}=\sigma_{b c}+u_{b c} e^{i \Delta t}$ where $i=1,2$ neglecting the fast oscillating terms [38]. Assuming $\Delta$ is much larger than any relaxation process, one can substitute with steady-state values of the fast varying terms: $\quad u_{a_{1} b} \cong\left[\Omega_{p u}\left(\rho_{a_{1} a_{1}}-\rho_{b b}\right)+\Omega_{p l}^{*} \rho_{a_{1} c}+\Omega_{p u} \rho_{a_{1} a_{2}}\right] / \Delta$, $u_{a_{2} b} \cong\left[\Omega_{p u}\left(\rho_{a_{2} a_{2}}-\rho_{b b}\right)+\Omega_{p l}^{*} \rho_{a_{2} c}+\Omega_{p u} \rho_{a_{2} a_{1}}\right] / \Delta, \quad$ and $u_{b c}=\left[\Omega_{p l}\left(\rho_{c c}-\rho_{b b}\right)+\Omega_{p u}^{*} \rho_{a_{1} c}+\Omega_{p u}^{*} \rho_{a_{2} c}\right] / \Delta$. Hence, the two-photon excitation can be described effectively in the density-matrix equations Eqs. 3b(a)-3b(i):

$$
\begin{aligned}
\dot{\sigma}_{a_{1} b}= & -\left(\Gamma_{a_{1} b}+i \delta_{1}\right) \sigma_{a_{1} b}+i \Omega_{a b}\left(\rho_{b b}-\rho_{a_{1} a_{1}}\right)-i \Omega_{b c}^{*} \rho_{a_{1} c}, \\
\dot{\sigma}_{a_{2} b}= & -\left(\Gamma_{a_{2} b}+i \omega_{21}+i \delta_{1}\right) \sigma_{a_{2} b}+i \Omega_{a b}\left(\rho_{b b}-\rho_{a_{2} a_{2}}\right)-i \Omega_{b c}^{*} \rho_{a_{2} c}, \\
\dot{\sigma}_{b c}= & -\Gamma_{b c} \sigma_{b c}+i \Omega_{b c}\left(\rho_{c c}-\rho_{b b}\right)+i \Omega_{a_{2} b}^{*} \rho_{a_{2} c}, \\
\dot{\rho}_{a_{1} c}= & \left(-\Gamma_{a_{1} c}-i \delta_{1}+i \frac{\left|\Omega_{p u}\right|^{2}-\left|\Omega_{p l}\right|^{2}}{\Delta}\right) \rho_{a_{1} c}+i \frac{\left|\Omega_{p u}\right|^{2}}{\Delta} \rho_{a_{2} c} \\
& +i \frac{\Omega_{p u} \Omega_{p l}}{\Delta}\left(\rho_{c c}-\rho_{a_{1} a_{1}}\right)+i \Omega_{a b} \sigma_{b c}-i \Omega_{b c} \sigma_{a_{1} b}, \\
\dot{\rho}_{a_{2} c}= & \left(-\Gamma_{a_{2} c}-i \omega_{21}-i \delta_{1}+i \frac{\left.\Omega_{p u}\right|^{2}-\left|\Omega_{p l}\right|^{2}}{\Delta}\right) \rho_{a_{2} c}+i \frac{\left|\Omega_{p u}\right|^{2}}{\Delta} \rho_{a_{1} c} \\
= & +i \frac{\Omega_{p u} \Omega_{p l}}{\Delta}\left(\rho_{c c}-\rho_{a_{2} a_{2}}\right)+i \Omega_{a b} \sigma_{b c}-i \Omega_{b c} \sigma_{a_{2} b}, \\
\dot{\rho}_{a_{1} a_{1}}= & -\gamma_{a_{1}} \rho_{a_{1} a_{1}}+\left(i \frac{\Omega_{p u} \Omega_{p l}}{\Delta} \rho_{c a_{1}}+i \Omega_{a b} \sigma_{b a_{1}}+\text { H.c. }\right), \\
\dot{\rho}_{a_{2} a_{2}}= & -\gamma_{a_{2}} \rho_{a_{2} a_{2}}+\left(i \frac{\Omega_{p u} \Omega_{p l}}{\Delta} \rho_{c a_{2}}+i \Omega_{a b} \sigma_{b a_{2}}+\text { H.c. }\right), \\
\dot{\rho}_{b b}= & -\gamma_{b} \rho_{b b}+\gamma_{a_{1}} \rho_{a_{1} a_{1}}+\gamma_{a_{2}} \rho_{a_{2} a_{2}}\left(i \Omega_{b c} \sigma_{c b}-i \Omega_{a b} \sigma_{b a_{1}}-i \Omega_{a b} \sigma_{b a_{2}}+\text { H.c. }\right), \\
& \rho_{a_{1} a_{1}}+\rho_{a_{2} a_{2}}+\rho_{b b}+\rho_{c c}=1,
\end{aligned}
$$

where the coherence $\rho_{a_{1} a_{2}}$ is neglected for simplicity. The dephasing rates are $\Gamma_{a_{i} b}=\frac{1}{2}\left(\gamma_{a_{i}}+\gamma_{b}\right)+\gamma_{\mathrm{col}}, \Gamma_{b c}=\frac{1}{2} \gamma_{b}+\gamma_{\mathrm{col}}$, $\Gamma_{a_{i} c}=\frac{1}{2} \gamma_{a_{i}}+\gamma_{\mathrm{col}}$, with $\gamma_{\mathrm{col}}$ being the collisional dephasing rate, and $\gamma_{i}$ is the spontaneous decay rate from level $i$. The Maxwell equations for generated Rabi frequencies read

$$
\begin{aligned}
& \frac{\partial \Omega_{a b}}{\partial z}+\frac{1}{c} \frac{\partial \Omega_{a b}}{\partial t}=i \eta_{a_{1} b} \rho_{a_{1} b}+i \eta_{a_{2} b} \rho_{a_{2} b}, \\
& \frac{\partial \Omega_{b c}}{\partial z}+\frac{1}{c} \frac{\partial \Omega_{b c}}{\partial t}=i \eta_{b c} \rho_{b c},
\end{aligned}
$$

where $\rho_{i j}$ is the slowly varying term of the atomic coherence, $\eta_{i j}=3 n_{a} \lambda_{i j}^{2} \gamma_{i} /(8 \pi), \lambda_{i j}$ is the transition wavelength, $\gamma_{i}$ is the spontaneous decay rate from level $i$, and $n_{a}$ is the number density of atomic oxygen. In the simulations, we assume that the upper levels $a_{1}$ and $a_{2}$ are comparably pumped and have the same decay rates. We set $\wp_{a b} \sim 1.38 \times 10^{-29} \mathrm{Cm}, \wp_{c c} \sim 3.80 \times 10^{-30} \mathrm{Cm}, \Delta \sim$ $6.11 \times 10^{15} \mathrm{rad} / \mathrm{fs}, \quad \delta_{1}=0.015 \times 10^{15} \mathrm{rad} / \mathrm{fs}, \quad \gamma_{a_{1}}=\gamma_{a_{2}}=$ $9.3 \times 10^{6} \mathrm{~s}^{-1}, \gamma_{b}=1.97 \times 10^{8} \mathrm{~s}^{-1}$, and the collisional dephasing rate $\gamma_{\mathrm{col}}=20 \mathrm{~ns}^{-1}$. The peak intensity of the pump pulse is $\left|E_{p}\right|^{2}=0.77 \times 10^{13} \mathrm{~W} / \mathrm{cm}^{2}$. We assume that there is small spontaneous fluctuations for the emission at $845 \mathrm{~nm}$ [32], and solve the Maxwell-Bloch equations for different O-atom concentrations $n_{a}$.

The simulated evolution of population differences $\rho_{a_{1} a_{1}}(t)-\rho_{b b}(t)$ and $\rho_{a_{2} a_{2}}(t)-\rho_{b b}(t)$ as a function of 

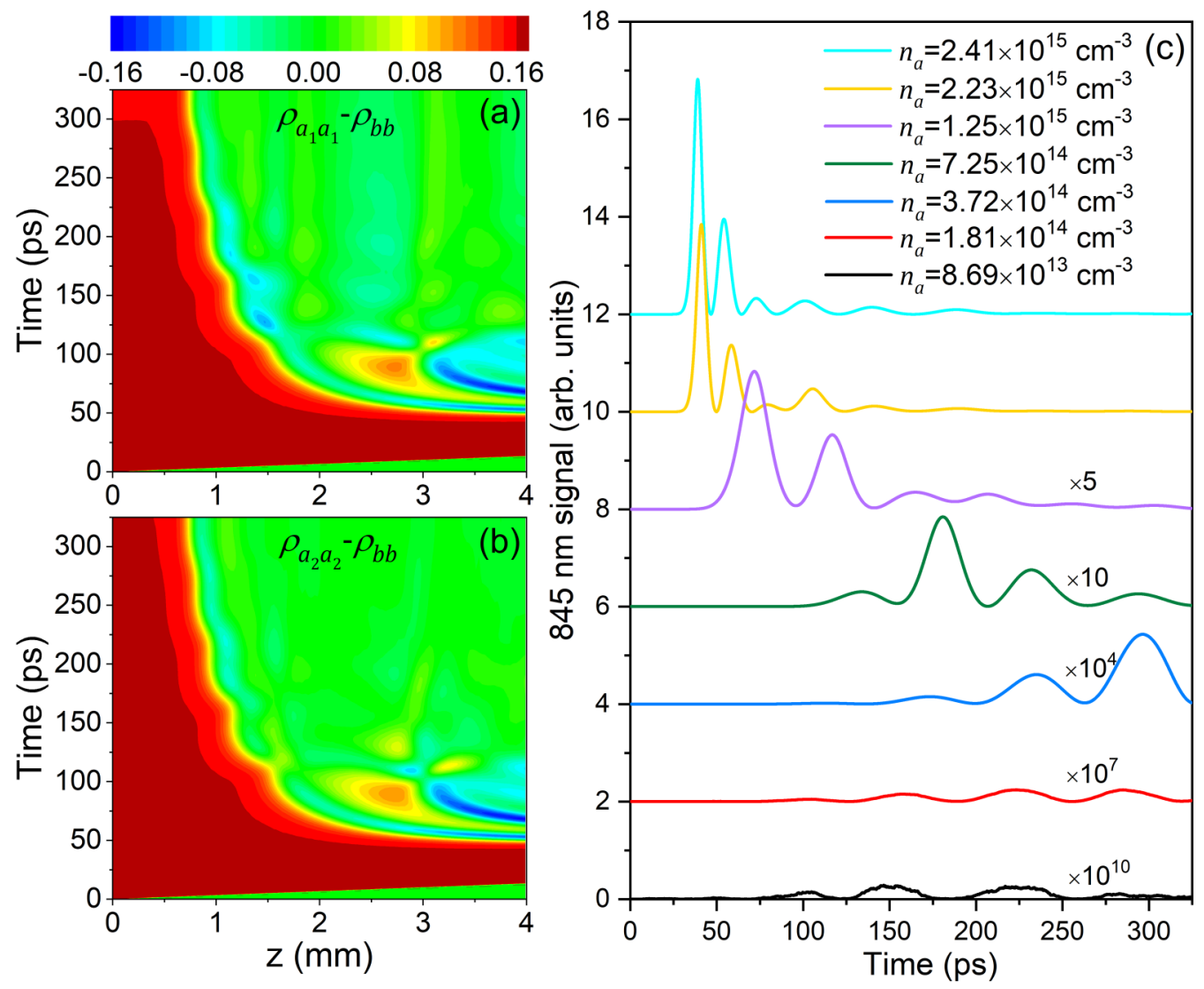

FIG. 4. (a) Simulated temporal profiles of 845 -nm signal for different concentrations. The curves for $n_{a}=1.25 \times 10^{15}, 7.25 \times 10^{14}, 3.72 \times$ $10^{14}, 1.81 \times 10^{14}$, and $8.69 \times 10^{13} \mathrm{~cm}^{-3}$ are magnified by $5,10,10^{4}, 10^{7}$, and $10^{10}$ times, respectively. Contour plots of the population difference (b) $\rho_{a_{1} a_{1}}-\rho_{b b}$ and (c) $\rho_{a_{2} a_{2}}-\rho_{b b}$ as a function of time and position for $n_{a}=2.41 \times 10^{15} \mathrm{~cm}^{-3}$.

time and position for $n_{a}=2.41 \times 10^{15} \mathrm{~cm}^{-3}$ is plotted in Figs. 4(a) and 4(b). The two upper levels have similar excitation and there are population inversions for both transitions due to the pump pulse, shown as the regions with positive values in Figs. 4(a) and 4(b). The evolution of the population difference exhibits the propagation effect and reabsorption and reemission processes are present, which can be seen by the rapid variations of the positive and negative values at the output position. In addition, we plot the numerical simulation results for the evolution of the 845-nm SF pulse in Fig. 4(c) to compare with the experimental results shown in Fig. 2(c). The results for low concentrations show more evident quantum-beating features. However, for the two highest concentrations, $n_{a}=2.41 \times 10^{15} \mathrm{~cm}^{-3}$ and $n_{a}=2.23 \times 10^{15} \mathrm{~cm}^{-3}$, the temporal ringing behavior is dominant in the cooperative emission process due to the propagation effect [30,32,39]. As the concentration gets lower, the period of beating becomes longer, approaching the value given by the frequency difference between the two upper levels of atomic oxygen. At the same time, the pulse shape changes and the intensity of the first peak drops. The variation trends of the pulse shape and intensity are in good agreement with the experimental results qualitatively. For low concentrations, the simulated SF signals decrease with the concentrations more rapidly compared to the experimental results. The rapid intensity reduction and extended delay in the simulation may come from the simplified modeling of the light-matter interaction in one dimension without considering the transverse effect. Three-dimensional Maxwell-Bloch simulations might be required for better agreement between the experiments and simulations.

\section{CONCLUSION}

In conclusion, we have reported experiments on the resonant two-photon excited lasing of atomic oxygen by deep-UV femtosecond laser pulses. The temporal profiles of the lasing pulse for different oxygen concentrations were measured. Our results show that the lasing accompanied by a quantumbeating signature is essentially oscillatory superfluorescence, suggesting the presence of atomic coherence. Numerical simulations based on the nonadiabatic Maxwell-Bloch equations reproduce well the above observations and confirm the critical role of atomic coherence. Our work shows fundamentals in the multiphoton excited lasing action in the flame and points towards important future applications in remote sensing and single-ended optical diagnostics.

\section{ACKNOWLEDGMENTS}

This research work was sponsored by the National Science Foundation for Young Scientists of China (Grant No. 12004147), the Knut and Alice Wallenberg Foundation (COCALD KAW2019.0084), the European Research 
Council (ERC Advanced Grant TUCLA 669466), the Swedish Research Council (VR), and the Swedish Foundation for Strategic Research (SSF, ITM17-0309). This work was also partially supported by the Fundamental Research
Funds for the Central Universities. L.Y. acknowledged the support from the Program for Professor of Special Appointment (Eastern Scholar) at Shanghai Institutions of Higher Learning.
[1] P. Polynkin and Y. Cheng, Air Lasing, Springer Series in Optical Sciences Vol. 208 (Springer, Berlin, 2018).

[2] L. Yuan, Y. Liu, J. Yao, and Y. Cheng, Recent advances in air lasing: A perspective from quantum coherence, Adv. Quantum Technol. 2, 1900080 (2019).

[3] P. Sprangle, J. Peñano, B. Hafizi, D. Gordon, and M. Scully, Remotely induced atmospheric lasing, Appl. Phys. Lett. 98, 211102 (2011).

[4] S. Mitryukovskiy, Y. Liu, P. Ding, A. Houard, and A. Mysyrowicz, Backward stimulated radiation from filaments in nitrogen gas and air pumped by circularly polarized $800 \mathrm{~nm}$ femtosecond laser pulses, Opt. Express 22, 12750 (2014).

[5] P. Ding, S. Mitryukovskiy, A. Houard, E. Oliva, A. Couairon, A. Mysyrowicz, and Y. Liu, Backward lasing of air plasma pumped by circularly polarized femtosecond pulses for the sake of remote sensing (black), Opt. Express 22, 29964 (2014).

[6] D. Kartashov, S. Ališauskas, A. Pugžlys, M. N. Shneider, and A. Baltuška, Theory of a filament initiated nitrogen laser, J. Phys. B: At., Mol. Opt. Phys. 48, 094016 (2015).

[7] S. Mitryukovskiy, Y. Liu, P. Ding, A. Houard, A. Couairon, and A. Mysyrowicz, Plasma Luminescence from Femtosecond Filaments in Air: Evidence for Impact Excitation with Circularly Polarized Light Pulses, Phys. Rev. Lett. 114, 063003 (2015).

[8] P. Ding, E. Oliva, A. Houard, A. Mysyrowicz, and Y. Liu, Lasing dynamics of neutral nitrogen molecules in femtosecond filaments, Phys. Rev. A 94, 043824 (2016).

[9] P. Ding, J. C. Escudero, A. Houard, A. Sanchis, J. Vera, S. Vicéns, Y. Liu, and E. Oliva, Nonadiabaticity of cavity-free neutral nitrogen lasing, Phys. Rev. A 96, 033810 (2017).

[10] R. Danylo, X. Zhang, Z. Fan, D. Zhou, Q. Lu, B. Zhou, Q. Liang, S. Zhuang, A. Houard, A. Mysyrowicz, E. Oliva, and Y. Liu, Formation Dynamics of Excited Neutral Nitrogen Molecules inside Femtosecond Laser Filaments, Phys. Rev. Lett. 123, 243203 (2019).

[11] M. Aldén, U. Westblom, and J. E. M. Goldsmith, Two-photonexcited stimulated emission from atomic oxygen in flames and cold gases, Opt. Lett. 14, 305 (1989).

[12] J. E. M. Goldsmith, Two-photon-excited stimulated emission from atomic hydrogen in flames, J. Opt. Soc. Am. B 6, 1979 (1989).

[13] M. Aldén, P.-E. Bengtsson, and U. Westblom, Detection of carbon atoms in flames using stimulated emission induced by two-photons laser excitation, Opt. Commun. 71, 263 (1989).

[14] S. Agrup, U. Westblom, and M. Aldén, Detection of atomic nitrogen using two-photon laser-induced stimulated emission: Application to flames, Chem. Phys. Lett. 170, 406 (1990).

[15] M. A. N. Georgiev, K. Nyholm, and R. Fritzon, Developments of the amplified stimulated emission technique for spatially resolved species detection in flames, Opt. Commun. 108, 71 (1994).

[16] U. Westblom, S. Agrup, M. Aldén, H. M. Hertz, and J. E. M. Goldsmith, Properties of laser-induced stimulated emission for diagnostic purposes, Appl. Phys. B 50, 487 (1990).
[17] M. S. Brown and J. B. Jeffries, Measurement of atomic concentrations in reacting flows through the use of stimulated gain or loss, Appl. Opt. 34, 1127 (1995).

[18] A. Dogariu, J. B. Michael, M. O. Scully, and R. B. Miles, Highgain backward lasing in air, Science 331, 442 (2011).

[19] A. J. Traverso, R. Sanchez-Gonzalez, L. Yuan, K. Wang, D. V. Voronine, A. M. Zheltikov, Y. Rostovtsev, V. A. Sautenkov, A. V. Sokolov, S. W. North, and M. O. Scully, Coherence brightened laser source for atmospheric remote sensing, Proc. Natl. Acad. Sci. USA 109, 15185 (2012).

[20] A. Laurain, M. Scheller, and P. Polynkin, Low-Threshold Bidirectional Air Lasing, Phys. Rev. Lett. 113, 253901 (2014).

[21] A. Dogariu and R. B. Miles, Three-photon femtosecond pumped backwards lasing in argon, Opt. Express 24, A544 (2016).

[22] P. Ding, M. Ruchkina, Y. Liu, M. Alden, and J. Bood, Femtosecond two-photon-excited backward lasing of atomic hydrogen in a flame, Opt. Lett. 43, 1183 (2018).

[23] M. Ruchkina, P. Ding, A. Ehn, M. Aldén, and J. Bood, Singleshot, spatially-resolved stand-off detection of atomic hydrogen via backward lasing in flames, Proc. Combust. Inst. 37, 1281 (2019).

[24] P. Ding, M. Ruchkina, Y. Liu, M. Alden, and J. Bood, Gain mechanism of femtosecond two-photon-excited lasing effect in atomic hydrogen, Opt. Lett. 44, 2374 (2019).

[25] P. Ding, M. Ruchkina, D. D. Cont-Bernard, A. Ehn, D. A. Lacoste, and J. Bood, Detection of atomic oxygen in a plasmaassisted flame via a backward lasing technique, Opt. Lett. 44, 5477 (2019).

[26] L. Yuan, B. H. Hokr, A. J. Traverso, D. V. Voronine, Y. Rostovtsev, A. V. Sokolov, and M. O. Scully, Theoretical analysis of the coherence-brightened laser in air, Phys. Rev. A 87, 023826 (2013).

[27] K. A. Rahman, V. Athmanathan, M. N. Slipchenko, S. Roy, J. R. Gord, Z. Zhang, and T. R. Meyer, Quantitative femtosecond, two-photon laser-induced fluorescence of atomic oxygen in high-pressure flames, Appl. Opt. 58, 1984 (2019).

[28] F. Migliorini, S. De Iuliis, F. Cignoli, and G. Zizak, How flat is the rich premixed flame produced by your mckenna burner? Combust. Flame 153, 384 (2008).

[29] G. P. Smith, D. M. Golden, M. Frenklach, N. W. Moriarty, B. Eiteneer, M. Goldenberg, C. T. Bowman, R. K. Hanson, S. Song, W. C. J. Gardiner, V. V. Lissianski, and Z. Qin, Gri 3.0 mechanism, http://combustion.berkeley.edu/gri-mech/ version30/text30.html.

[30] M. Gross and S. Haroche, Superradiance: An essay on the theory of collective spontaneous emission, Phys. Rep. 93, 301 (1982).

[31] F. T. Arecchi and E. Courtens, Cooperative phenomena in resonant electromagnetic propagation, Phys. Rev. A 2, 1730 (1970).

[32] J. C. MacGillivray and M. S. Feld, Theory of superradiance in an extended, optically thick medium, Phys. Rev. A 14, 1169 (1976). 
[33] A. Kumarakrishnan, S. Chudasama, and X. Han, Collisioninduced superfluorescence, J. Opt. Soc. Am. B 22, 1538 (2005).

[34] J. V. Thompson, C. W. Ballmann, H. Cai, Z. Yi, Y. V. Rostovtsev, A. V. Sokolov, P. Hemmer, A. M. Zheltikov, G. O. Ariunbold, and M. O. Scully, Pulsed cooperative backward emissions from non-degenerate atomic transitions in sodium, New J. Phys. 16, 103017 (2014).

[35] F. Marin, C. Fort, M. Prevedelli, M. Inguscio, G. M. Tino, and J. Bauche, Hyperfine structure and isotope shift of the $3 s-3 p$ transitions of atomic oxygen, Z. Phys. D: At., Mol. Clusters 25, 191 (1993).
[36] A. F. H. van Gessel, S. C. van Grootel, and P. J. Bruggeman, Atomic oxygen TALIF measurements in an atmosphericpressure microwave plasma jet with in situ xenon calibration, Plasma Sources Sci. Technol. 22, 055010 (2013).

[37] M. O. Scully and M. S. Zubairy, Quantum Optics (Cambridge University Press, Cambridge, UK, 1997).

[38] Nonlinear Optics, edited by R. W. Boyd, 3rd ed. (Academic, Burlington, 2008), p. v.

[39] J. J. Maki, M. S. Malcuit, M. G. Raymer, R. W. Boyd, and P. D. Drummond, Influence of collisional dephasing processes on superfluorescence, Phys. Rev. A 40, 5135 (1989). 